\title{
A SURVEY ON THE ENVELOPING METHOD OF SOME ONE-DIMENSIONAL NON-AUTONOMOUS DISCRETE PERIODIC POPULATION MODELS
}

\section{RAFAEL LUÍS ${ }^{1}$ and ELIAS RODRIGUES ${ }^{2}$}

\author{
1,2University of Madeira \\ Funchal, Madeira \\ Portugal \\ e-mail: rafael.luis.madeira@gmail.com \\ elias@uma.pt \\ ${ }^{1}$ Center for Mathematical Analysis, \\ Geometry, and Dynamical Systems \\ University of Lisbon \\ Lisbon \\ Portugal
}

\begin{abstract}
For some one-dimensional discrete-time autonomous population models, local stability implies global stability of the positive equilibrium point. One of the known techniques is the enveloping method. In this paper, we present a survey on the enveloping method to study global stability of single periodic population models. In the other words, we present the conditions in which "individual enveloping" implies "periodic enveloping" in some one-dimensional periodic population models.
\end{abstract}

2010 Mathematics Subject Classification: Primary 37C75; Secondary 39A23, 39A30, 39A60.

Keywords and phrases: periodic population models, local stability, global stability, enveloping, applications.

Received April 28, 2016

(C) 2016 Scientific Advances Publishers 


\section{Introduction}

One-dimensional models are an appropriate mathematical tool to model the behaviour of populations with non-overlapping generations. This subject has been intensely investigated by different researchers.

An autonomous population model is a difference equation of the form

$$
x_{n}=f\left(x_{n}\right), \quad n \in \mathbb{Z}_{0}^{+},
$$

where the map $f$ is a continuous function from the nonnegative reals to the nonnegative reals and there is a unique positive number $x^{*}$, the equilibrium point, such that

$$
\begin{aligned}
& f(0)=0, \\
& f(x)>x \text { for } 0<x<x^{*}, \\
& f(x)=x \text { for } x=x^{*} \\
& f(x)<x \text { for } x>x^{*}
\end{aligned}
$$

Furthermore, the map $f$ is bounded on $\left[0, x^{*}\right]$. Notice that these conditions correspond to the usual assumptions in population dynamics.

After normalization, we can always assume that $x^{*}=1$. In [8], we can find a complete study for the local properties of $x^{*}$. In general, it is much more complicated to investigate the global stability of $x^{*}$. The following result found in [10, Corollary 2.4] and [19, Theorem 2.1, pp. 47] gives a condition on global stability.

Theorem 1.1. Let $x^{*}$ be a fixed point of a continuous map $f$ on the compact interval $[a, b]$. Then $x^{*}$ is globally asymptotically stable relative to the interval $(a, b)$ if and only if $f^{2}(x)>x$ for $x<x^{*}$ and $f^{2}(x)<x$ for $x>x^{*}$, for all $x \in(a, b)$. 
In the other words, a continuous population model is globally stable if and only if it has no cycles of minimal period 2. This result was noticed much earlier by Coppel in 1955 [1].

Theorem 1.2. Let $I=[a, b] \subseteq \mathbb{R}$ and $f: I \rightarrow I$ be a continuous map. If $f$ has no points of prime period two, then every orbit under the map f converges to a fixed point.

Unfortunately, this global stability condition may be difficult to test. Moreover, it seems that there is no obvious connection between the local stability conditions and the global stability conditions.

In a series of papers $[2,3,4,5]$, Cull and his collaborators embarked in the theory that "enveloping" implies global stability. A function $h(x)$ envelops a function $f(x)$ if and only if

(i) $h(x)>f(x)$ for all $x \in\left(0, x^{*}=1\right)$;

(ii) $h(x)<f(x)$ for $x>x^{*}=1$ such that $h(x)>0$ and $f(x)>0$.

Combining this definition with Theorem 1.1 and Theorem 1.2, one has the following result:

Theorem 1.3 ([5]). If $f(x)$ is enveloped by $g(x)$, and $g(x)$ is globally stable, then $f(x)$ is globally stable.

Hence, the enveloping function plays a central rule in this theory. In [4], Cull presented the following result concerning the enveloping function.

Theorem 1.4. Let $h(x)$ be a monotone decreasing function which is positive on $\left(0, x_{h}>1\right)$ and so that $h(h(x))=x$. Assume that $f(x)$ is a continuous function such that

$$
\begin{aligned}
& h(x)>f(x) \text { on }(0,1), \\
& h(x)<f(x) \text { on }\left(1, x_{h}\right),
\end{aligned}
$$




$$
\begin{aligned}
& f(x)>x \text { on }(0,1), \\
& f(x)<x \text { on }(1, \infty), \\
& f(x)>0 \text { whenever } x>1 .
\end{aligned}
$$

Then for all $x>0, \lim _{n \rightarrow \infty} f^{n}(x)=1$.

The preceding theorem shows the importance of the enveloping function in global stability. So, the challenge will be to find the appropriate enveloping. Surprisingly or not, the following Möbius transformation may help in finding the appropriate enveloping.

Theorem 1.5 ([4]). If $f(x)$ is enveloped by a linear fractional function of the form $h(x)=\frac{1-\alpha x}{\alpha-(2 \alpha-1) x}, \alpha \in[0,1)$, then $f(x)$ is globally stable.

Since there are many options for enveloping in the preceding theorem, it is necessary to adjust each particular model with the enveloping in the set of parameters. Hence, this task is not easy.

Eduardo Liz [18] tried to simplify this process. He studied enveloping function for models of the form $x_{n+1}=x_{n}+f\left(x_{n}\right)$ and uses Schwarzian derivative of $f$. He was able to characterize when local stability implies global stability in certain one-dimensional population models. His results are based in the following proposition:

Proposition 1.6 ([18]). Let $f$ to be a population model defined as before and suppose that $f$ is a $C^{3}$ map that has at most one critical point $x_{c}$. If $\left|f^{\prime}\left(x^{*}\right)\right| \leq 1$ and $S f(x)<0$ for all $x \neq x_{c}$, then $x^{*}$ is a globally stable fixed point of $f$, where $S f$ is the Schwarzian derivative of $f$ given by

$$
S f(x)=\frac{f^{\prime \prime \prime}(x)}{f^{\prime}(x)}-\frac{3}{2}\left(\frac{f^{\prime \prime}(x)}{f^{\prime}(x)}\right)^{2}
$$

Later on, Rubió-Massegú and Mañosa in [20] brings up the importance of the enveloping function since the enveloping implies the existence of a global Lyapunov function. Thus, the global asymptotically stability can be seen as a consequence of an invariant principle. 
Recently, Wrigth [24] extended the previous idea of enveloping to periodic discrete models. It has been shown that under certain conditions the periodic system is globally stable, namely, if the sequence of maps have the same common fixed point and there exists a decreasing enveloping $h$ with $h \circ h(x)=x$ such that $h$ envelops all the maps, then the periodic equation is globally stable.

Our main objective in this paper is to provide a survey on the theory of enveloping method used to show global stability in one-dimensional periodic population models.

In Section 2, we present some preliminaries concerning the general theory of non-autonomous periodic difference equations. In the next section, we present the principal results in the field of periodic equations, i.e., we give the condition for which individual enveloping implies periodic enveloping for mappings. The next section is devoted to applications. We illustrate the results in several well known models in population dynamics as is the cases of the periodic Ricker model, the periodic generalized Beverton-Holt model and the periodic logistic model. A mixing population models are studied as well.

We believe that the tools presented in this survey may be used as a tutorial in some undergraduate project. It gives the primary ideas of global stability and can be viewed as an open door to study global stability in other kind of models, either in one dimension or in higher dimension as well.

\section{Periodic Systems}

A difference equation is called non-autonomous if it is governed by the rule

$$
x_{n+1}=f_{n}\left(x_{n}\right), \quad n \in \mathbb{Z}^{+},
$$

where $x \in X$ and $X$ is a topological space. Here the orbit of a point $x_{0}$ is generated by the composition of the sequence of maps

$$
f_{0}, f_{1}, f_{2}, \ldots
$$


Explicitly,

$$
\begin{aligned}
x_{1}= & f_{0}\left(x_{0}\right), \\
x_{2}= & f_{1}\left(x_{1}\right)=f_{1} \circ f_{0}\left(x_{0}\right), \\
& \vdots \\
x_{n+1}= & f_{n} \circ f_{n-1} \circ \ldots \circ f_{1} \circ f_{0}\left(x_{0}\right),
\end{aligned}
$$

If the sequence of maps is periodic, i.e., $f_{n+p}=f_{n}$, for all $n=0,1,2, \ldots$ and some positive integer $p>1$, then we talk about nonautonomous periodic difference equations. Systems where the sequence of maps is periodic, model population with fluctuation habitat, and they are commonly called periodically forced systems.

Throughout this paper, we work with non-autonomous periodic difference equation in which $p$ is the minimal period of Equation (2.1) and $X=\mathbb{R}$.

Notice that the non-autonomous periodic difference equation (2.1) does not generate a discrete (semi)dynamical system [14] as it may not satisfy the (semi)group property. One of the most effective ways of converting the non-autonomous difference equation (2.1) into a genuine discrete (semi)dynamical system is the construction of the associated skew-product system as described in a series of papers by Elaydi and Sacker $[11,13,14,15]$. It is noteworthy to mention that this idea was originally used to study non-autonomous differential equations by Sacker and Sell [22].

Definition 2.1. An ordered set of points $C_{r}=\left\{\bar{x}_{0}, \bar{x}_{1}, \ldots, \bar{x}_{r-1}\right\}$ is an $r$-periodic cycle in $X$ if

$$
f_{(i+n r) \bmod p}\left(\bar{x}_{i}\right)=\bar{x}_{(i+1) \bmod r}, \quad n \in \mathbb{Z}^{+} .
$$

In particular,

$$
f_{i}\left(\bar{x}_{i}\right)=\bar{x}_{i+1}, \quad 0 \leq i \leq r-2,
$$


and

$$
f_{t}\left(\bar{x}_{t \bmod r}\right)=\bar{x}_{(t+1) \bmod r}, \quad r-1 \leq t \leq p-1 .
$$

It should be noted that the $r$-periodic cycle $C_{r}$ in $X$ generates an $s$-periodic cycle on the skew-product $X \times Y\left(Y=\left\{f_{0}, f_{1}, \ldots, f_{p-1}\right\}\right)$ of the form

$$
\widehat{C}_{s}=\left\{\left(\bar{x}_{0}, f_{0}\right),\left(\bar{x}_{1}, f_{1}\right), \cdots,\left(\bar{x}_{(s-1) \bmod r}, f_{(s-1) \bmod p}\right)\right\},
$$

where $s=l c m[r, p]$ is the least common multiple of $r$ and $p$.

To distinguish these two cycles, the $r$-periodic cycle $C_{r}$ on $X$ is called an $r$-geometric cycle (or simply $r$-periodic cycle when there is no confusion), and the $s$-periodic cycle $\widehat{C}_{s}$ on $X \times Y$ is called an $s$-complete cycle. Notice that either $r<p$, or $r=p$ or $r>p$.

Define the composition operator $\Phi$ as follows:

$$
\Phi_{n}^{i}=f_{n+i-1} \circ \ldots \circ f_{i+1} \circ f_{i} .
$$

When $i=0$ we write $\Phi_{n}^{0}$ as $\Phi_{n}$.

As a consequence of the above remarks it follows that the $s$-complete

cycle $\widehat{C}_{s}$ is a fixed point of the composition operator $\Phi_{s}^{i}$. In the other words, we have that

$$
\Phi_{s}^{i}\left(\bar{x}_{i \bmod r}\right)=\bar{x}_{i \bmod r} .
$$

If the sequence of maps $\left\{f_{i}\right\}, i \geq 0$ is a parameter family of maps one-toone in the parameter, then by [9], we have that $\bar{x}_{i \bmod p}$ is a fixed point of $\Phi_{p}$.

\section{Enveloping in Periodic Models}

In this section, we present the idea of enveloping method in periodic single species of population models. 
In population dynamics, it is common to work with a parameter family of maps. If we are working with a certain population model, we can always rescale the positive fixed point to $x^{*}=1$. Hence, under this scenario, a parameter family of maps will have the same positive fixed point. Having in mind this idea, we point out a first assumption:

H1. Let $\mathcal{F}=\left\{f_{0}, f_{1}, f_{2}, \ldots\right\}$ to be a set of $C^{1}$ population models such that $f_{i}(1)=1$, for all $i=0,1,2, \ldots$. Assume that the composition

$$
\Phi_{p}(x)=f_{p-1} \circ \ldots \circ f_{1} \circ f_{0}(x),
$$

is continuous in a subset of the nonnegative reals. Further, in order to guaranty periodicity of the equation

$$
x_{n+1}=f_{n}\left(x_{n}\right),
$$

we also require that the maps on $\mathcal{F}$ are periodic with period $p$, i.e., $f_{n+p}=f_{n}$, for all $n$.

A natural question arises in the field of periodic difference equations: is the composition of population models a population model?

The answer of this question, in general, is negative, it depends on the individual maps. In Figure 1, we present a concrete example, where

$$
f_{0}(x)=\left\{\begin{array}{l}
4 x \text { if } 0 \leq x<0.6 \\
-3.5 x+4.5 \text { if } 0.6 \leq x<1, \\
\frac{1}{x} \text { if } x>1,
\end{array}\right.
$$

and

$$
f_{1}(x)=\left\{\begin{array}{l}
3 x \text { if } 0 \leq x<0.5 \\
-x+2 \text { if } 0.5 \leq x<1 \\
\frac{1}{x} \text { if } x>1
\end{array}\right.
$$




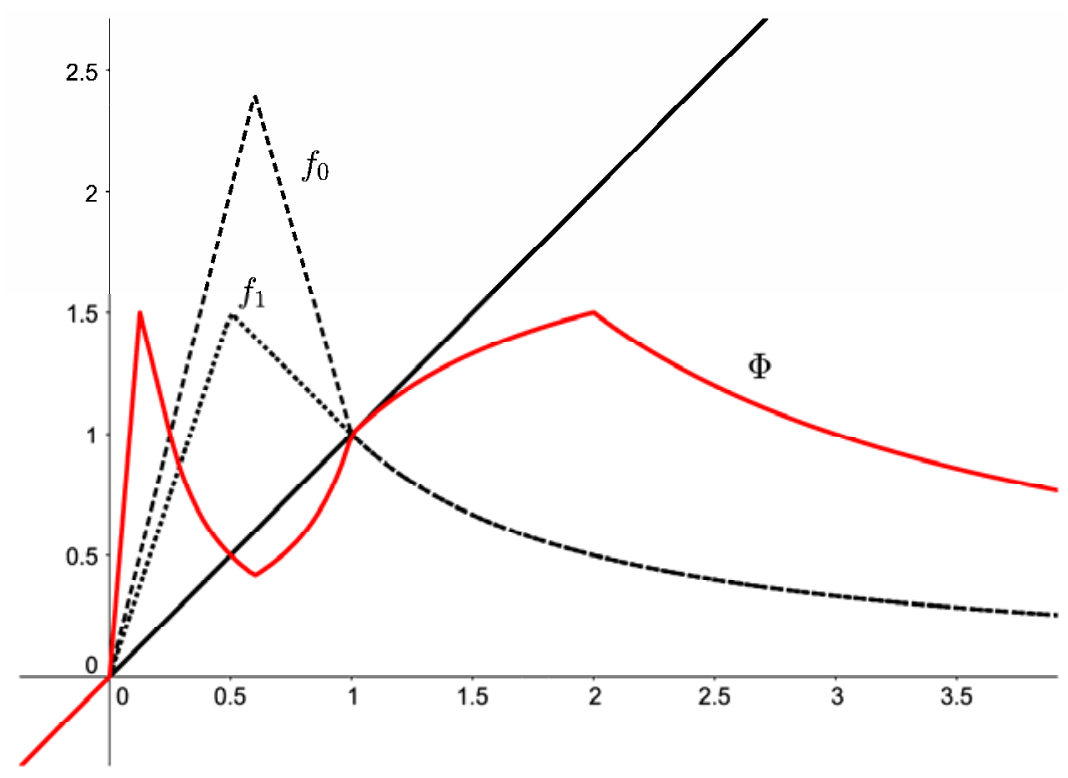

Figure 1. This example shows that the composition of population models may not be a population model.

Observe that the maps of $\mathcal{F}$ are increasing in certain interval. Hence, one can show the following proposition:

Proposition 3.1. Under hypothesis H1, the composition map $\Phi_{p}(x)$ is increasing in $\left(0, c_{\Phi}\right)$, for certain positive value $c_{\Phi}$. Moreover, there exists $x_{\Phi}<x_{\Phi}^{*}$ such that $\Phi_{p}(x)>f_{i}(x)$, for all $x \in\left(0, x_{\Phi}\right), i \in\{0,1, \ldots, p-1\}$ with $\Phi_{p}\left(x_{\Phi}^{*}\right)=x_{\Phi}^{*}$.

Proof. It follows from our hypothesis that each individual map $f_{i}$ is increasing on $\left(0, c_{i}\right)$, for some $c_{i}>0$ (eventually the map $f_{i}$ can be increasing in all the domain). Since the composition of monotone mappings is a monotone map, it follows that there exists $c_{\Phi}>0$, the minimum of the critical values of $\Phi_{p}$, such that $\Phi_{p}(x)$ is an increasing function on $\left(0, c_{\Phi}\right)$. 
Since each one of the individual maps $f_{i}$ is increasing on $\left(0, c_{i}\right)$ and the origin is an unstable fixed point of all the maps, we have that $f_{i}^{\prime}(0)>1$, for all $i \in\{0,1, \ldots, p-1\}$. Taking the derivative of the composition map, we have

$$
\begin{aligned}
\Phi_{p}^{\prime}(x) & =f_{p-1}^{\prime}\left(\Phi_{p-1}(x)\right) \times f_{p-2}^{\prime}\left(\Phi_{p-2}(x)\right) \times \ldots \times f_{1}^{\prime}\left(f_{0}(x)\right) \times f_{0}^{\prime}(x) \\
& =\prod_{i=0}^{p-1} f_{i}^{\prime}\left(\Phi_{i}(x)\right) .
\end{aligned}
$$

Hence, at the origin, we have

$$
\Phi_{p}^{\prime}(0)=\prod_{i=0}^{p-1} f_{i}^{\prime}\left(\Phi_{i}(0)\right)>>f_{i}^{\prime}(0), \quad i \in\{0,1, \ldots, p-1\} .
$$

This implies that there exists a positive number $x_{\Phi}<x_{\Phi}^{*}$ such that

$$
\Phi_{p}(x)>f_{i}(x), \quad \text { for all } \quad x \in\left(0, x_{\Phi}\right), \quad i \in\{0,1, \ldots, p-1\} .
$$

The next assumption will be in the enveloping function. First, let us observe the following example where it is shown that "individual enveloping" do not implies "periodic enveloping".

Example 3.2. Let $f_{0}(x)=x e^{1.5(1-x)}$ and $f_{1}(x)=x e^{1.2(1-x)}$ and assume that $f_{n+2}=f_{n}$, for all $n$. It is clear that $f_{0}, f_{1} \in \mathcal{F}$ and $x_{n+1}=$ $f_{n}\left(x_{n}\right)$ is a 2-periodic difference equation.

Each one of the maps $f_{0}$ and $f_{1}$ is enveloped by the map $g(x)=x e^{2(1-x)}$. The map $g(x)$ is a globally asymptotically stable population model since it is enveloped by the decreasing fractional function $h(x)=2-x$ (for more details, see [5]). Moreover, from Theorem 1.3 , one can conclude that the individual maps $f_{i}(x), i=0,1$ are globally stable since they are envelop by $g(x)$. 
To study the dynamics of the 2-periodic difference equation, we study the dynamics of the map

$$
\Phi_{2}(x)=f_{1} \circ f_{0}(x)=x e^{2.7-1.5 x-1.2 x e^{1.5(1-x)}} .
$$

Plotting the graph of $\Phi_{2}(x)$ one can conclude that there exists a positive value $a<1=x_{\Phi}^{*}$ such that

$$
\Phi_{2}(x)>x e^{2(1-x)}, \text { for all } x \in(0, a)
$$

and

$$
\Phi_{2}(x)<x e^{2(1-x)} \text {, for all } x \in(a, 1)
$$

Consequently, $\Phi_{2}(x)$ is not enveloped by $g(x)$. However, the individual maps $f_{0}$ and $f_{1}$ are enveloped by $g(x)$. Hence, we can not conclude stability of $\Phi_{2}(x)$ from the individual enveloping $g(x)$. In Subsection 4.1, we will show that $\Phi_{2}(x)$ is also enveloped by $h(x)=2-x$ and consequently from Theorem 1.5 it is globally stable.

We remind that the main goal in this field is to find certain class of maps where individual enveloping implies periodic enveloping. Due the preceding examples, we have to guarantee: (i) the composition of population models is a population model and (ii) the individual enveloping is also an enveloping for the composition map, i.e., we have to guarantee that the composition map has two fixed points, the origin and a positive fixed point $x_{\Phi}^{*}, \Phi_{p}(x)>x$ if $x \in\left(0, x_{\Phi}^{*}\right)$ and $\Phi(x)_{p}<x$ if $x>x_{\Phi}^{*}$, and there exits an enveloping for a sequence of the individual maps $f_{i}$ that envelops the composition map. These observations motivates the second assumption: 
H2. There exits a decreasing envelop $h$ such that $h(x)$ envelops all the maps in the set $\mathcal{F}$ and $h \circ h(x)=x$.

The following proposition may help us in the construction of an envelop. We will omit the prove since the result follows directly by symmetry.

Proposition 3.3. Let $h$ to be an enveloping of a population model $f$ in the conditions of hypothesis $\mathbf{H 2}$. Consider the graph of $f$ and the curve, $S_{f}$, obtained from the graph of $f$ by symmetry with respect to the diagonal $y=x$. Then, the graph of $h$ lies between the graph of $f$ and the curve $S_{f}$ everywhere, with the exception of the fixed point $x^{*}=1$.

We are now ready to present the main result in this field. It states the conditions in which individual enveloping implies periodic enveloping. An alternative proof may be found in [24].

Theorem 3.4. Under hypotheses $\mathbf{H} 1$ and $\mathbf{H 2}$, the composition map $\Phi_{p}(x)$ is a globally asymptotically stable population model.

Proof. The prove follows by induction. We will show the result for the composition of two maps $(p=2)$ and the proof for the general case $(p>2)$ may be obtained in a suitable way.

Combining $\mathbf{H} 1$ and $\mathbf{H} 2$ it follows from Theorem 1.4 that $f_{i}(x)$, $i=0,1,2, \ldots$ is a sequence of globally asymptotically stable population models.

Since $f_{i}(0)=0$ and $f_{i}(1)=1$, for all $i=0,1,2, \ldots$ it follows that $\Phi_{p}(0)=0$ and $\Phi_{p}(1)=1$. The uniqueness of $x_{\Phi}^{*}=1$ will follow in the following arguments.

Let us first study the composition map $\Phi_{2}(x)=f_{1} \circ f_{0}(x)$. Since $\Phi_{2}(1)=1$ we split the prove into two cases: $\mathbf{I}-0<x<1$ and II $-x>1$. 
Case I. If $0<x<1$, either $f_{0}(x)>1$ or $x<f_{0}(x)<1$ (recall that $f_{0}(x)>x$ when $\left.x \in(0,1)\right)$.

If $f_{0}(x)>1$, then $f_{1} \circ f_{0}(x)=f_{1}\left(f_{0}(x)\right)<f_{0}(x)<h(x)$. Since $h$ is decreasing and $f_{1}(y)>h(y)$, for all $y>1$, we have that

$$
x=h \circ h(x)<h \circ f_{0}(x)<f_{1} \circ f_{0}(x) .
$$

If $x<f_{0}(x)<1$, then $h \circ f_{0}(x)<h(x)$. But, since $f_{0}(x)<1$, we have $f_{1} \circ f_{0}(x)<h \circ f_{0}(x)<h(x)$. On the other hand, $x<f_{0}(x)<f_{1} \circ f_{0}(x)$.

Case II. Let $x>1$ and assume first $0<f_{0}(x)<1$. This implies that $f_{1} \circ f_{0}(x)>f_{0}(x)>h(x)$. In order to prove that $f_{1} \circ f_{0}(x)<x$, we notice that

$$
x=h \circ h(x)>h \circ f_{0}(x)>f_{1} \circ f_{0}(x) .
$$

When $x>f_{0}(x)>1$, we have that $h \circ f_{0}(x)>h(x)$ and consequently $f_{1} \circ f_{0}(x)>h \circ f_{0}(x)>h(x)$. Since $f_{0}(x)>1$, it follows that $x>f_{0}(x)>$ $f_{1} \circ f_{0}(x)$.

We have shown that $\Phi_{2}(x)$ is a population model and the enveloping function $h$ envelops the composition map $\Phi_{2}$. Consequently, from Theorem 1.4, the map $\Phi_{2}(x)$ is a globally asymptotically stable population model.

It will be beneficial, in certain cases, to have the contrapositive of this theorem which we write in the following corollary:

Corollary 3.5. If $f_{0}, f_{1}, \ldots, f_{p-1}$ are population models and the composition map $\Phi_{p}$ is not a population model, then it can not exist an enveloping $h$, in the conditions of hypothesis $\mathbf{H 2}$, such that henvelops all the individual population models. 


\section{Applications}

In this section, we illustrate how the results apply to some onedimensional periodic population models. We present a family of Ricker maps, a family of Beverton-Holt models, quadratic models, mixing models, and harvesting models.

\subsection{Ricker model}

Let us consider the periodic difference equation given by the following equation:

$$
x_{n+1}=R_{n}\left(x_{n}\right) \text {, }
$$

where the sequence of maps $R_{n}(x)$ is given by

$$
R_{n}(x)=x e^{r_{n}(1-x)}, \quad r_{n}>0, \quad n=0,1,2, \ldots
$$

The local stability condition $0<r_{n} \leq 2, n \in\{0,1, \ldots\}$ of each individual population model $R_{i}(x)$ implies global stability of $R_{i}(x)$, since each one of the individual maps $R_{n}(x), n \in\{0,1, \ldots\}$ is enveloped by $h(x)=2-x$, which is a fractional decreasing function with $\alpha=1 / 2$ (see Theorem 1.5). In the other words, $x^{*}=1$ is a globally asymptotically stable fixed point of $R_{n}(x), n \in\{0,1, \ldots\}$. Notice that $h \circ h(x)=x$. Thus, hypothesis $\mathbf{H} 2$ is satisfied. 


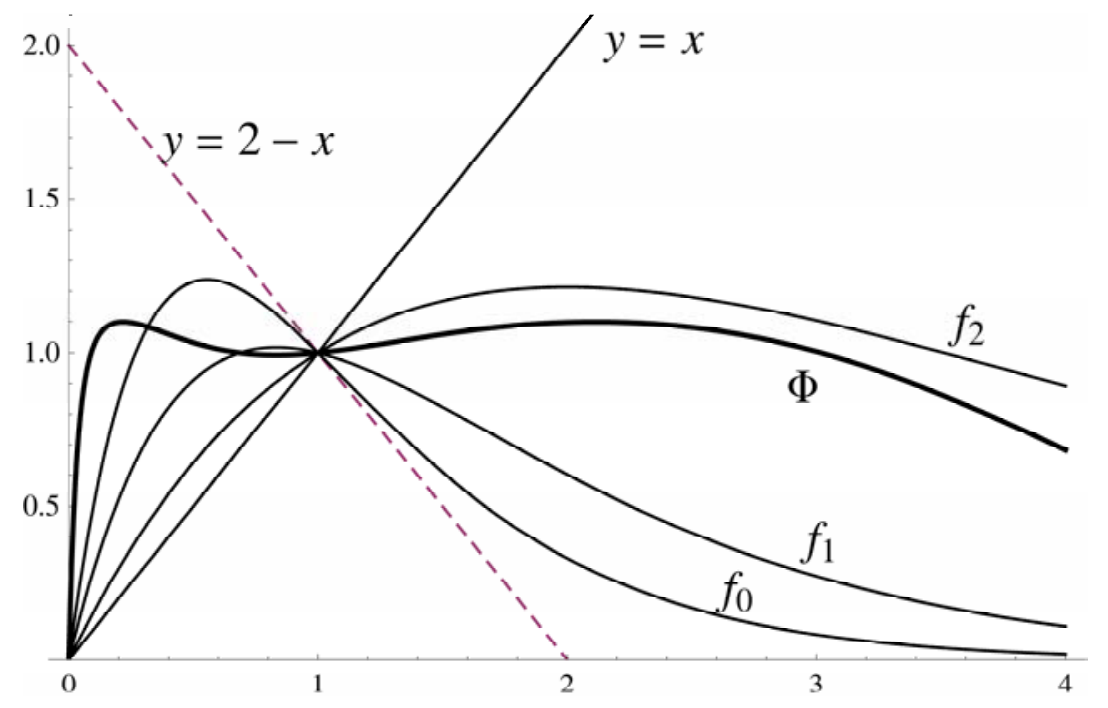

Figure 2. An illustration of the "individual enveloping" (tiny curves) and the "composition enveloping" (solid curve) in the one-parameter family of a Ricker type map. In this case, individual enveloping implies periodic enveloping and consequently the global stability of the positive fixed point in the periodic equation.

In order to have periodicity, we require that $R_{n+p}=R_{n}$, for all $n=0,1,2, \ldots$, i.e., the sequence of parameters satisfies $r_{n}=r_{n \bmod p}$ for all $n$. It is clear that the composition map

$$
\Phi_{p}(x)=R_{p-1} \circ \ldots \circ R_{1} \circ R_{0}(x),
$$

is continuous in $\mathbb{R}_{0}^{+}$. Consequently, hypothesis $\mathbf{H} \mathbf{1}$ is satisfied. Hence, from Theorem 3.4, it follows that $\Phi_{p}(x)$ is a globally asymptotically stable population model, i.e., the $p$-periodic Ricker difference equation is globally stable whenever $r_{n} \in(0,2], n=0,1,2, \ldots$.

Hence, in this family of population models, individual enveloping implies periodic enveloping. 
In Figure 2 is represented a concrete example where $r_{0}=1.8$, $r_{1}=1.2$, and $r_{2}=0.5$. The composition map $\Phi_{3}=R_{2} \circ R_{1} \circ R_{0}$ is represented by the solid curve. The dashed line is the enveloping function while the tiny curves are the individual population models.

Before ending this example, we notice that $\prod_{i=0}^{p-1}\left|1-r_{i}\right|<1$ is the stability condition for the fixed point $x^{*}=1$ under the action of the composition map $\Phi_{p}$.

Finally, we should mention that Sacker [21] used a different method to show global stability of a similar periodic Ricker type model given by

$$
x_{n+1}=x_{n} e^{r_{n}-x_{n}},
$$

in the parameter region $0<r_{n} \leq 2, n=0,1,2, \ldots, p-1$.

\subsection{Generalized Beverton-Holt model}

Let $x_{n+1}=B_{n}\left(x_{n}\right), n=0,1,2, \ldots$, where the map $B_{n}$ is given by

$$
B_{n}(x)=\frac{\mu_{n} x}{1+\left(\mu_{n}-1\right) x^{c_{n}}} .
$$

Assume that $\mu_{n}>1$ and $0<c_{n} \leq 2$, for all $n=0,1,2, \ldots$.

The individual population map $B_{n}(x)$ has two fixed point, the origin and a positive fixed point given by $x^{*}=1$. It is easily shown that the origin is an unstable fixed point since $\left|B_{n}^{\prime}(0)\right|=\mu_{n}>1$, for all $n=0,1,2, \ldots$. The condition of local stability of the positive fixed point is given by $\mu_{n}\left(c_{n}-2\right) \leq c_{n}$, for all $n=0,1,2, \ldots$ This condition implies global stability since each individual map $B_{n}(x), n=0,1,2, \ldots$ is enveloped by

$$
h(x)=\frac{1}{x},
$$

which is a decreasing fractional function with $h \circ h(x)=x$. Hence, H2 is satisfied. 
Let us assume now the periodicity of the map $B_{n}$ by taking $\mu_{n+q}=\mu_{n}$ and $c_{n+r}=c_{n}$ for some $q, r=1,2,3, \ldots$ This implies that $B_{n+p}=B_{n}$, where $p=\operatorname{lcm}(q, r)$. From the fact that $r_{n}>1$, for all $n$, it follows that $1+\left(r_{n}-1\right) x^{c_{n}}>0$ whenever $x \in \mathbb{R}^{+}$. Hence, the composition of the Beverton-Holt models is well defined and thus we have the continuity of the composition. Consequently, $\mathbf{H 1}$ is satisfied. It follows from Theorem 3.4 that

$$
\Phi_{p}(x)=B_{p-1} \circ \ldots \circ B_{1} \circ B_{0}(x),
$$

is a globally asymptotically stable population model. Consequently, the $p$-periodic Beverton-Holt equation $x_{n+1}=B_{n}\left(x_{n}\right), B_{n+p}=B_{n}, n=0,1,2, \ldots$ is globally stable whenever $\mu_{n}>1$ and $0<c_{n} \leq 2$.

Notice that the condition of stability of the positive fixed point of $\Phi_{p}$ is given by

$$
\prod_{i=0}^{p-1}\left|1+\left(\mu_{i}-1\right)\left(1-c_{i}\right)\right|<\prod_{i=0}^{p-1} \mu_{i}
$$

We should mention that when $c_{n}=1$, we have the classical Beverton-Holt model. In a series of papers $[6,7,10,11,12,13,14,15,16$, $17,23]$, the authors used a different method to study the global stability of the positive periodic cycle.

In order to have a complete study of this model, it remains to study the cases where $c_{n}>2$ for all $n$, or a possible mixing case in the parameters $c_{n}$, i.e., some of the parameters are less or equal than 2 and others are greater than 2. Under these scenarios, the individual enveloping is

$$
h(x)=\left\{\begin{array}{l}
\frac{1}{2} \quad \text { if } c_{n} \leq 2 \\
\frac{c_{n}-1-\left(c_{n}-2\right) x}{c_{n}-2-\left(c_{n}-3\right) x} \text { if } c_{n}>2 .
\end{array}\right.
$$


We point out that, in certain cases is possible to find a common enveloping for all the $p$ individual maps and hence global stability of the periodic equation. However, this is not the general case, as we show in the following concrete example.

Let $\mu_{0}=1.1, \mu_{1}=7, c_{0}=7.5$ and $c_{1}=2.3$. Hence, the individual maps are given by

$$
f_{0}(x)=\frac{1.1 x}{1+0.1 x^{0.5}} \quad \text { and } \quad f_{1}(x)=\frac{7 x}{1+6 x^{2.3}} .
$$

Both $f_{0}$ and $f_{1}$ are globally asymptotically stable population models with respect to the positive fixed point since they are enveloped by

$$
h_{0}(x)=\frac{6.5-5.5 x}{5.5-4.5 x} \quad \text { and } h_{1}(x)=\frac{1.3-0.3 x}{0.3+0.7 x}
$$

respectively (see Figure 3).

As is clearly shown in Figure 3 the composition map $\Phi_{2}(x)=f_{1} \circ f_{0}(x)$ has 3 positive fixed points and consequently can not be globally stable. Clearly, $\Phi_{2}$ is not a population model. From Corollary 3.5 follows that it can not exist a decreasing enveloping $h$, with $h \circ h(x)=x$, such that $h$ envelops simultaneously $f_{0}$ and $f_{1}$.

Notice that, if that enveloping exits, then from Proposition 3.3, it lies between the graphs of $f_{0}$ and $f_{1}$ and the respective curves, $S_{f_{0}}$ and $S_{f_{1}}$, obtained from $f_{0}$ and $f_{1}$ by symmetry with respect to the diagonal $y=x$. As it is clearly shown in Figure 3, there exits an interval ]$a, b[\subset] 1.5,1.6[$ where such enveloping fails. 


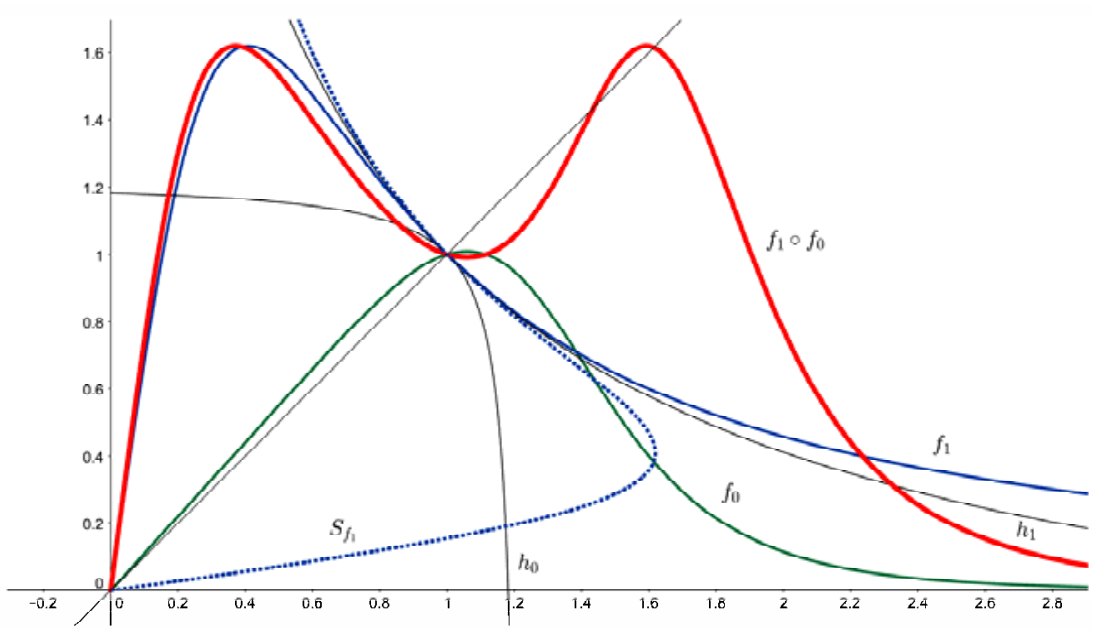

Figure 3. An example showing that there is no global stability in the 2-periodic Beverton-Holt model, when $\mu_{0}=1.1, \mu_{1}=7, c_{0}=7.5$ and $c_{1}=2.3$. In this case is not possible to find a decreasing enveloping that envelops simultaneously the individual population models $f_{0}$ and $f_{1}$.

\subsection{Mixing models: Beverton-Holt acting with Ricker model}

Let us now consider that the sequence of maps is given by

$$
f_{n}(x)=\left\{\begin{array}{l}
x e^{r_{n}(1-x)} \text { if } n \text { is even, } \\
\frac{\mu_{n} x}{1+\left(\mu_{n}-1\right) x^{c_{n}}} \text { if } n \text { is odd, }
\end{array}\right.
$$

where $0<r_{n} \leq 2, \mu_{n}>1$, and $0<c_{n} \leq 1$ for all $n$. Assume the periodicity of the parameters, i.e., $r_{n+q}=r_{n}, \mu_{n+r}=\mu_{n}$, and $c_{n+s}=c_{n}$ for some positive integer $q, r$, and $s$. Hence, the equation $x_{n+1}=f_{n}\left(x_{n}\right)$ is $p$-periodic with $p=\operatorname{lcm}(q, r, s)$. Clearly, $f_{n}(x) \in \mathcal{F}$ and consequently H1 is satisfied.

Now, from Subsection 4.1, the sequence of maps $f_{2 n}(x), n=0,1, \ldots$ is enveloped by the decreasing function $h(x)=2-x$. It is easy to see that

$$
f_{2 n+1}(x)=\frac{\mu_{2 n+1} x}{1+\left(\mu_{2 n+1}-1\right) x^{c_{2 n+1}}}
$$


$\mu_{n}>1$ and $0<c_{n} \leq 1, n=1,3,5, \ldots$ is also enveloped by $h(x)=2-x$. To see this observe that $f_{2 n+1}(1)=h(1), h$ is decreasing and $f_{2 n+1}(x)$ is increasing since

$$
f_{2 n+1}^{\prime}(x)=\frac{\mu_{2 n+1}+\mu_{2 n+1}\left(\mu_{2 n+1}-1\right)\left(1-c_{2 n}\right) x^{c_{2 n+1}}}{\left(1+\left(\mu_{2 n+1}-1\right) x^{c_{2 n+1}}\right)^{2}}>0 .
$$

This implies that $\mathbf{H 2}$ is satisfied. Consequently, from Theorem 3.4 follows that

$$
\Phi_{p}(x)=f_{p-1} \circ \ldots \circ f_{1} \circ f_{0}(x),
$$

is a globally asymptotically stable population model. Hence, the $p$ periodic difference equation $x_{n+1}=f_{n}\left(x_{n}\right), n=0,1,2, \ldots$ is globally stable.

Finally, we determine the stability condition under composition operator, which is given by $\left|\Phi_{p}^{\prime}(1)\right|<1$. A forward computation shows that

$$
\Phi_{p}^{\prime}(1)=\left\{\begin{array}{l}
\prod_{n=0}^{\frac{p}{2}-1} \frac{\left(1-r_{2 n}\right)\left(\mu_{2 n+1}-\left(\mu_{2 n+1}-1\right) c_{2 n+1}\right)}{\mu_{2 n+1}} \text { if } p \text { is even, } \\
\left(1-r_{p-1}\right) \prod_{n=0}^{\frac{p-1}{2}-1} \frac{\left(1-r_{2 n}\right)\left(\mu_{2 n+1}-\left(\mu_{2 n+1}-1\right) c_{2 n+1}\right)}{\mu_{2 n+1}} \text { if } p \text { is odd. }
\end{array}\right.
$$

Notice that a similar approach can be done in the case that we consider the even sequence of maps a Beverton-Holt type and the odd sequence of maps a Ricker-type model.

\subsection{Exponential and rational}

Let us consider the non-autonomous difference equation

$$
x_{n+1}=\frac{\left(1+a_{n} e^{b_{n}}\right) x_{n}}{1+a_{n} e^{b_{n} x_{n}}},
$$

where $0<b_{n} \leq 2$ and $a_{n}>0$, for all $n=0,1,2, \ldots$. This equation can be defined by the following map: 


$$
f_{n}(x)=\frac{\left(1+a_{n} e^{b_{n}}\right) x}{1+a_{n} e^{b_{n} x}}
$$

It is easy to check that the conditions of local stability at the fixed point $x^{*}=1$

$$
a_{n}\left(b_{n}-2\right) e^{b_{n}} \leq 2
$$

implies global stability since each map $f_{n}$ is enveloped by $h(x)=2-x$ in $\mathbb{R}_{0}^{+}$.

Let $a_{n+q}=a_{n}$ and $b_{n+r}=b_{n}$, for all $n=0,1,2, \ldots$ Then the sequence of maps is $p$-periodic, where $p=\operatorname{lcm}(q, r)$, i.e., the nonautonomous equation $x_{n+1}=f_{n}\left(x_{n}\right)$ is $p$-periodic. Clearly, the periodic composition map $\Phi_{p}(x)$ is continuous in $\mathbb{R}_{0}^{+}$since $1+a_{n} e^{b_{n} x} \neq 0$ for all $x \geq 0$ and $0<b_{n} \leq 2$ and $a_{n}>0$, for all $n=0,1,2, \ldots$.

Since $\mathbf{H 1}$ and $\mathbf{H} 2$ are satisfied, from Theorem 3.4 follows the global stability in the non-autonomous periodic equation.

\subsection{Quadratic model}

Let $x_{n+1}=L_{n}\left(x_{n}\right)$, where $L_{n}(x)=x\left(1+\mu_{n}(1-x)\right), x \in I_{n}=\left[0,1+\frac{1}{\mu_{n}}\right]$, for all $n=0,1,2, \ldots$ The local stability condition for each individual population model $L_{n}(x), n \in\{0,1, \ldots, p-1\}$ is given by $0<\mu_{i} \leq 2$. Since the fractional function $h(x)=\frac{4-3 x}{3-2 x}$ envelops each map $L_{n}(x)$, $n \in\{0,1, \ldots, p-1\}$ it follows that $L_{n}(x)$ is a globally asymptotically stable population model whenever $0<\mu_{i} \leq 2$. Notice that $h \circ h(x)=x$. Hence $\mathbf{H} 2$ is satisfied.

Let us now assume the periodicity of the difference equation by taking $L_{n+p}=L_{n}$, for all $n=0,1,2, \ldots$, i.e., the sequence of 
parameters are $p$-periodic. In order to guarantee the continuity of the composition operator, we construct the interval $J$ as follows. Let $I$ to be an interval given by

$$
I=\bigcap_{n=0}^{p-1}\left[0,1+\frac{1}{\mu_{n}}\right] .
$$

The interval $J$ is defined by

$$
J=\bigcap_{i=0}^{p-1} L_{n}(I)
$$

Clearly, $J \supseteq[0,1], \Phi_{p}(1)=1$ and $\Phi_{p}(x)$ is continuous for all $x \in J$. Hence $\mathbf{H 1}$ is satisfied. It follows from Theorem 3.4 that

$$
\Phi_{p}(x)=L_{p-1} \circ \ldots \circ L_{1} \circ L_{0}(x), \quad x \in J,
$$

is a globally asymptotically stable population model whenever $0<\mu_{n} \leq 2$, $n=0,1,2, \ldots$. Consequently, $x^{*}=1$ is a globally stable fixed point of the $p$-periodic difference equation $x_{n+1}=L_{n}\left(x_{n}\right)$.

\subsection{Beverton-Holt with harvesting}

Let us consider the difference equation $x_{n+1}=f_{n}\left(x_{n}\right)$, where the sequence of maps $f_{n}$ is given by

$$
f_{n}(x)=\frac{r_{n} x}{1+\left(r_{n}-1\right) x}-c_{n} x(x-1), r_{n}>1,0<c_{n}<1, \text { for all } n
$$

In this model, we are taking $x$ in the interval

$$
I_{n}=\left[0, \frac{\left(r_{n}-2\right) \sqrt{c_{n}}-\sqrt{r_{n}\left(r_{n}\left(4+c_{n}\right)-4\right)}}{2\left(r_{n}-1\right) \sqrt{c_{n}}}\right] .
$$

Clearly $f_{n}(x)$ is a population model for all $x \in I_{n}$. Moreover, the extinction fixed point is unstable since $f_{n}^{\prime}(0)=r_{n}+c_{n}>1$ for all $n=0,1,2, \ldots$ The local stability condition for $x^{*}=1$ is given by 


$$
0<c_{n}<\frac{1+r_{n}}{r_{n}} \text {, for all } n
$$

Since the fractional function $h(x)=\frac{11-8 x}{8-5 x}$ envelops each individual map $f_{n}(x), n=0,1,2, \ldots, \quad$ it follows that $f_{n}(x)$ is a globally asymptotically stable population model whenever $x$ belongs to the interior of $I_{n}$ and $0<c_{n}<\frac{1+r_{n}}{r_{n}}, n=0,1,2, \ldots$. Notice that $h \circ h(x)$ $=x$.

Let us now assume the periodicity of the parameters by taking $r_{n+q}=r_{n}$, and $c_{n+r}=c_{n}$ for all $n=0,1,2, \ldots$. Hence, the sequence of maps is $p$-periodic, where $p=l c m(q, r)$. Define the interval $J$ as follows:

$$
J=\bigcap_{n=0}^{p-1} f_{n}(I), \quad \text { where } \quad I=\bigcap_{n=0}^{p-1} I_{n} .
$$

Since $\mathbf{H} 1$ and $\mathbf{H} \mathbf{2}$ are satisfied, it follows from Theorem 3.4 that

$$
\Phi_{p}(x)=f_{p-1} \circ \ldots \circ f_{1} \circ f_{0}(x), \quad x \in J
$$

is a globally asymptotically stable population model whenever $0<c_{n}<$ $\frac{1+r_{n}}{r_{n}}$, for all $n=0,1,2, \ldots$, i.e., $x^{*}=1$ is a globally stable fixed point of the $p$-periodic Beverton-Holt equation with harvesting.

\section{Final Remarks}

In this survey, we have presented the conditions when individual enveloping implies periodic enveloping for certain periodic population models. In the other words, if a sequence of population models is enveloped by a common decreasing function $h$ such that $h(h(x))=x$, then the periodic equation is globally stable with respect to the positive equilibrium. 
In population dynamics, this observation stats that, if each one of the individual population is globally stable with respect to the positive equilibrium, then the population with fluctuation habitat is also globally stable with respect to the positive equilibrium. A several examples are given in order to illustrate the results.

Hence, in the case of certain periodic forced systems, local stability implies global stability with respect to the positive fixed point.

\section{References}

[1] W. A. Coppel, The solution of equations by iteration, Mathematical Proceedings of the Cambridge Philosophical Society 51(01) (1955), 41-43.

[2] P. Cull, Stability in one-dimensional models, Scientiae Mathematicae Japonicae 58 (2003), 349-357.

[3] P. Cull, Enveloping implies global stability, In L. Allen, B. Aulbach, S. Elaydi and R. Sacker, editors, Difference Equations and Discrete Dynamical Systems, pages 170-181, Hackensack, NJ, 2005. World Scientific.

[4] P. Cull, Population models: Stability in one dimension, Bulletin of Mathematical Biology 69(3) (2007), 989-1017.

[5] P. Cull, K. Walsh and J. Wherry, Stability and instability in one-dimensional population models, Scientiae Mathematicae Japonicae Online pages 29-48, e-2008.

[6] J. Cushing and S. Henson, Global dynamics of some periodically forced, monotone difference equations, Journal of Difference Equations and Applications 7 (2001), 859-872.

[7] J. Cushing and S. Henson, A periodically forced Beverton-Holt equation, Journal of Difference Equation and Applications 8 (2002), 1119-1120.

[8] S. Elaydi, Discrete Chaos: With Applications in Science and Engineering, Chapman and Hall/CRC, Second Edition, 2008.

[9] S. Elaydi, R. Luis and H. Oliveira, Towards a theory of periodic difference equations and its application to population dynamics, In Mauricio Matos Peixoto, Alberto Adrego Pinto and David A. Rand, editors, Dynamics, Games and Science I, pages 287-321. DYNA 2008, in Honor of Maurcio Peixoto and David Rand, University of Minho, Braga, Springer, March 2011. Series: Springer Proceedings in Mathematics, Vol. 1.

[10] S. Elaydi and R. Sacker, Basin of attraction of periodic orbits of maps in the real line, Journal of Difference Equations and Applications 10 (2004), 881-888.

[11] S. Elaydi and R. Sacker, Global stability of periodic orbits of nonautonomous difference equations and populations biology, J. Differential Equations 208 (2005), 258-273. 
[12] S. Elaydi and R. Sacker, Global stability of periodic orbits of nonautonomous difference equations in population biology and the Cushing-Henson conjectures, In Saber Elaydi, Gerasimos Ladas, Bernd Aulbach and Ondrej Dosly, editors, Proceedings of the Eighth International Conference on Difference Equations and Applications, pages 113-126, Chapman and Hall/CRC, 2005.

[13] S. Elaydi and R. Sacker, Nonautonomous Beverton-Holt equations and the CushingHenson conjectures, Journal of Difference Equations and Applications 11(4-5) (2005), 337-346.

[14] S. Elaydi and R. Sacker, Skew-product Dynamical Systems: Applications to Difference Equations, Proceedings of the Second Annual Celebration of Mathematics, United Arab Emirates, 2005.

[15] S. Elaydi and R. Sacker, Periodic difference equations, population biology and the Cushing-Henson conjectures, Mathematical Biosciences 201 (2006), 195-207.

[16] V. Kocic, A note on the nonautonomous Beverton-Holt model, Journal of Difference Equation and Applications 11(4-5) (2005), 415-422.

[17] R. Kon, A note on attenuant cycles of population models with periodic carrying capacity, Journal of Difference Equation and Applications 10(8) (2004), 791-793.

[18] E. Liz, Local stability implies global stability in some one-dimensional discrete single-species models, Discrete and Continuous Dynamical Systems-Series B 7(1) (2007), 191-199.

[19] A. Sharkovsky, Yu. Maistrenko and E. Romanenko, Difference Equations and their Applications, Kluwer Academic Publishers, London, 1993.

[20] J. Rubió-Massegú and Víctor Mañosa, On the enveloping method and the existence of global Lyapunov functions, Journal of Difference Equations and Applications 13(11) (2007), 1029-1035.

[21] R. Sacker, A note on periodic Ricker map, Journal of Difference Equations and Applications 13(1) (2007), 89-92.

[22] R. Sacker and J. Sell, Lifting properties in skew-product flows with applications to differential equations, AMS Memoirs 11(190), 1977.

[23] S. Stevic, A Short Proof of the Cushing-Henson conjecture, Discrete Dynamics in Nature and Society, Hindawi Publishing Corporation, 2006.

[24] J. Wright, Periodic systems of population models and enveloping functions, Computers and Mathematics with Applications 66 (2013), 2178-2195. 